\title{
MAGNETIC DESIGN CONSIDERATIONS TO IMPROVE NON-LINEAR CHARACTERISTICS OF INDUCTIVELY COUPLED POWER TRANSFER SYSTEMS
}

\author{
Dariusz Kacprzak, Jan K. Sykulski * \\ The University of Auckland, Department of Electrical and Computer Engineering, \\ 38 Princess street, Auckland, New Zealand, e-mail: d.kacprzak@auckland.ac.nz \\ *University of Southampton, School of Electronics and Computer Science, \\ Southampton SO17 1BJ, United Kingdom, e-mail: J.K.Sykulski@soton.ac.uk
}

Abstract - The paper discusses the magnetic features of various pick-up arrangements of Inductively Coupled Power Transfer systems. The performance of the standard monorail system containing an E-pickup can be improved by using S- or Z-shaped magnetic circuits. Moreover, in the case of the Z-pickup, it is possible to benefit from the positive mutual coupling if an alternate-direction configuration is adopted.

\section{INTRODUCTION}

The Inductively Coupled Power Transfer (ICTP) technology has made significant advances recently in applications where no mechanical contact is essential or advantageous. Today ICPT systems are widely used in low and high power applications in both domestic and industrial markets. A popular function of the ICPT technology is to supply power to monorail transportation systems used in clean rooms or automotive production lines.

A typical ICTP system is shown in Fig. 1; it comprises a current carrying Litz-wired track and a pickup [1]. The pickup is attached to a moving platform and is designed to travel along the primary track. Standard monorail ICPT systems are often based on an E-pickup, named after the shape of the ferromagnetic core. Figure 2 shows an example E-pickup in a monorail ICPT system.

Although the E-pickup configuration is quite practical and easy to install on the monorail track, it has several crucial disadvantages. In particular, it has an inefficient magnetic design resulting in a rather low power to volume of ferrite ratio $(\mathrm{S} / \mathrm{v})$ [2]. When high power transfer is required, the monorail platform often uses two E-pickups to double the power. However, a multi-pickup formation of E-pickups produces the so-called negative mutual coupling between neighbouring pickups, resulting in a reduction of total power by more than $10 \%$.

\section{COMPUTING POWER OF ICPT SYSTEMS}

In order to predict computationally the power transferred by the pickup system, two separate Finite Element Method (FEM) analyses are performed. The first calculation aims at finding the open circuit voltage $V_{o c}$, and the second at determining the short circuit current $I_{\mathrm{sc}}$. $V_{\text {oc }}$ can be obtained from a numerical model where the conductivity of the pickup's coil is set to zero. $I_{\text {sc }}$ is usually computed from a model where a representative conductivity of the Litz-wired coil is substituted. The following formulae are then used to determine the uncompensated power [3]:

$$
\begin{gathered}
V_{o C(m s)}=N \cdot B_{o v(m s)} \cdot A_{B \perp} \cdot \omega \\
I_{s c(m s)}=\frac{J_{(m s)} \cdot A_{I \perp}}{N} \\
S=Q V_{o c} I_{s c}
\end{gathered}
$$

where: $B_{a v .(r m s)}$ is an average value of the magnetic flux density in the ferrite (under the coil area), $J_{(r m s)}$ is an average value of the current density in the coil, $A_{B \perp}$ is the crosssectional area of the ferromagnetic material under the coil (perpendicular to $B_{a v .(m s)}$ ), $A_{I \perp}$ is the cross-sectional area of the coil (perpendicular to $J_{(r m s)}$ ), and $Q$ is the quality factor introduced in [4].

An experiment was carried out to verify the concept of the FEM analysis as well as to find the correspondence between the model and the physical measurements [3]. It was found that the numerical results agree very well with the experiment with errors not exceeding $5 \%$.

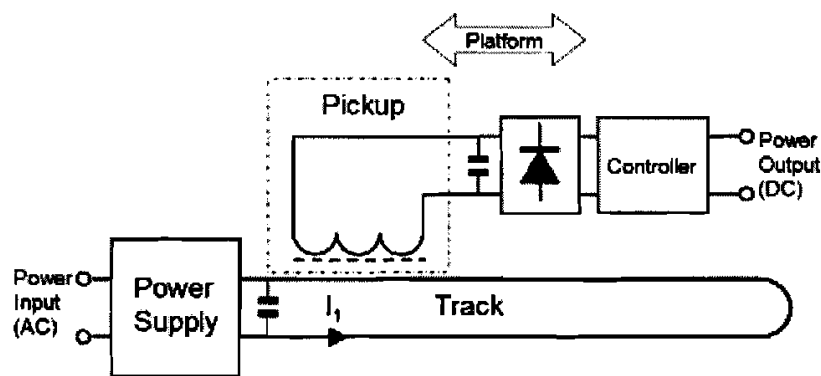

Fig. 1. Diagram of an Inductively Coupled Power Transfer system

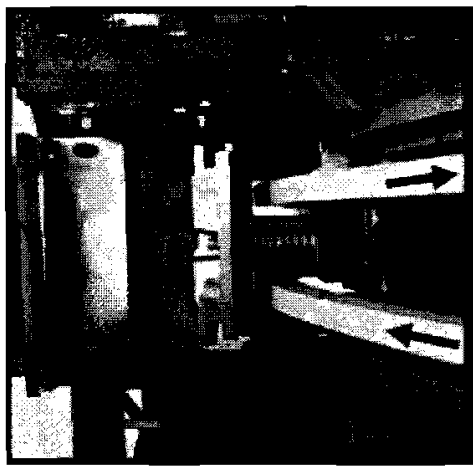

Fig. 2. An E-pickup in a monorail ICPT system 


\section{ALTERNATIVE PICKUP CONFIGURATIONS}

Alternative S- and Z-shaped pickups have been proposed to replace standard E-pickups to improve the performance of monorail ICPT systems [2]. Figure 3 shows the shapes of all analyzed pickups, as well as a simplified flow of the magnetic flux. Accurate FEM images of the magnetic field distributions are not presented as they are difficult to read.

The S-pickup performs extremely well. It can be characterized by an excellent power to volume of ferrite ratio, nearly twice the value for an equivalent E-pickup, as the Spickup transfers $95 \%$ more power for the same volume of ferrite. This excellent power transfer ability of the S-pickup is a consequence of changing the position of the block $\mathrm{Fl}$ as indicated in Fig. $3(\mathrm{a}, \mathrm{b})$. Whereas in the E-pickup F1 conducts the magnetic flux through its entire length, thus presenting a significant reluctance to the flux, in the S-pickup the block F1 absorbs the magnetic field via its large sidesurface, thus introduces a much lower reluctance.

The Z-pickup is strongly related to the S-pickups, but possesses $40 \%$ less ferrite, thus it is cheaper and lighter (refer to Fig. 4). It has a relatively high power to ferrite volume ratio as shown in Fig. 5. The results were obtained for a $10 \mathrm{kHz}$ ICPT system. Overall the Z-pickup delivers slightly less power than the E-pickup, but the difference is negligible.

One of the most interesting features of the Z-pickup is its performance in a multi-pickup configuration. A novel alternate-direction arrangement is shown in Fig. 6. In this configuration two Z-pickups produce positive mutual coupling between them indicated by $+\phi_{\mathrm{m}}$. As a result the power of this system is higher when pickups are closer as shown in Fig. 7. Thus the inherent non-linearity of the output characteristic is improved and the signal almost insensitive to the presence of the neighbouring units.

(a)

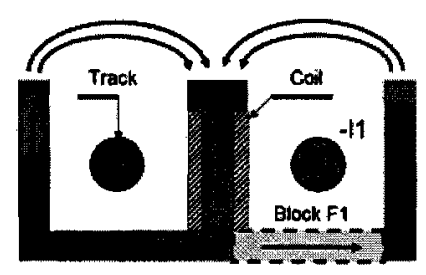

(b)

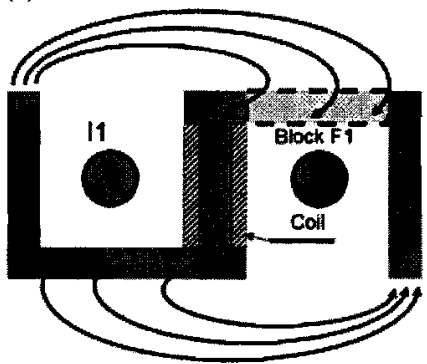

Fig. 3. Analysed pickups (a) E-pickup (b) S-pickup
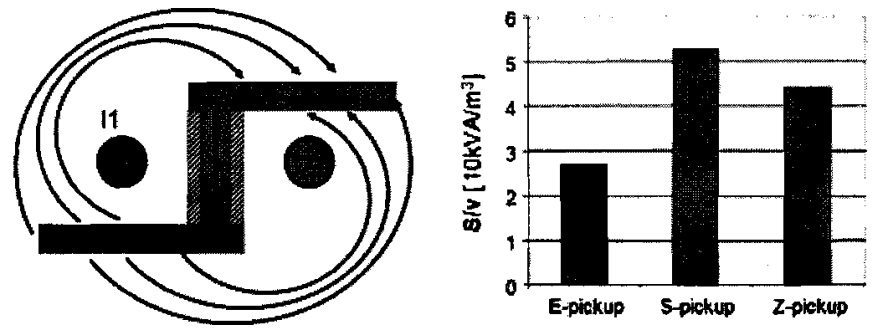

Fig. 4. Z-pickup

Fig. 5. Power to volume ration

\section{CONCLUSIONS}

Careful analysis of magnetic field distributions is important in the design of modern power pick-ups to optimise the performance of magnetic circuits and benefit from available field reinforcement due to positive coupling. In this presentation an S-pickup has been suggested as an alternative to the common E-pickup as providing almost double the power output for a given volume of ferrite, at no increase in cost. The Z-pickup, on the other hand, offers the possibility of reduced weight at similar performance; moreover, its nonlinearity may be improved in multi-pickup configurations by applying an alternate direction concept.

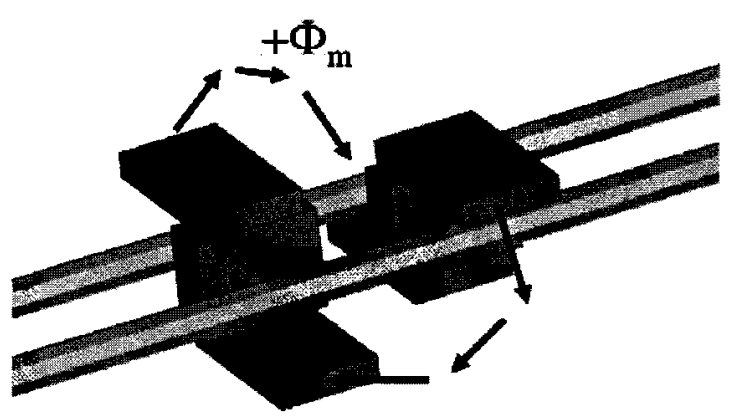

Fig. 6. Multi pickup configuration

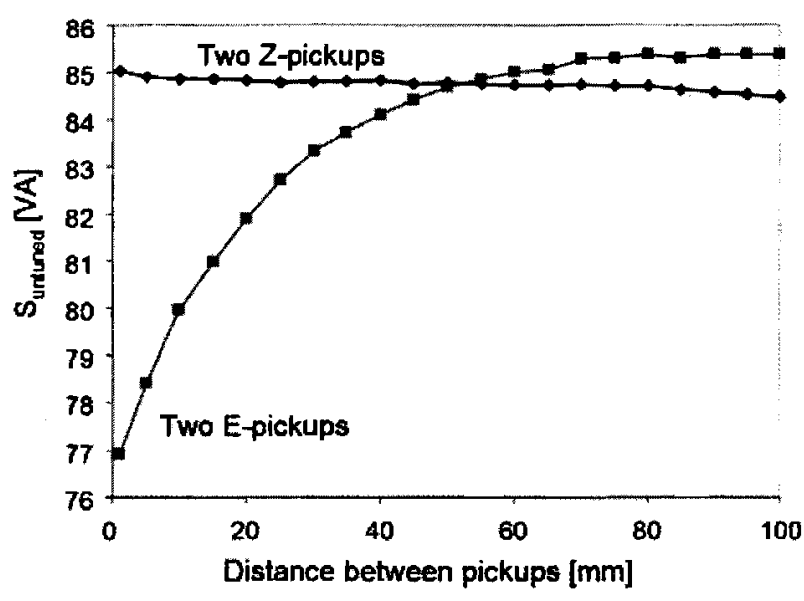

Fig. 7. Power of a multi-pickup configuration

\section{REFERENCES}

[1] A.W. Green, J.T. Boys, "10kHz inductively coupled power transfer concept and control", Power Electronics and Variable-Speed Drives, Conference Publication No. 399, (C) IEE, 1994.

[2] D. Kacprzak, "A new concept of asymmetrical pickups for monorail inductively coupled power transfer systems", Conference Publication Intermag 2006, No. CU-06, 2006.

[3] D. Kacprzak, G.A. Covic., J.T. Boys, "An improved magnetic design for inductively coupled power transfer system pickups", Proceeding of the $7^{\text {th }}$ International Power Engineering Conference IPEC 2005, 2005.

[4] J.T. Boys, A.P. Hu, G.A. Covic, "Critical $Q$ analysis of a current-fed resonant converter for ICPT applications", Electronics Letters, Vol. 36, No. 17,2000 